\title{
fluage, dilatance ou compressibilité des roches autour des puits verticaux et de forages pétroliers
}

\author{
N. CRISTESCU \\ Département de mécanique \\ Université de Bucarest
}

\section{Résumé}

En forage pétrolier ou pour un puits de mine on se pose le problème important de déterminer si la roche autour du puits devient dilatante ou si elle devient compressible après le forage. Une loi de comportement élasto/viscoplastique capable de décrire la dilatance ou la compressibilité des roches est formulée. On donne la distribution des contraintes, des déformations et des déplacements autour du puits. Le fluage de la roche, c'est-à-dire la convergence très lente des parois du puits ainsi que la relaxation des contraintes qui accompagne le fluage, sont aussi étudiées avec ce modèle. On détermine ensuite les circonstances (c'est-à-dire l'état primaire, généralement arbitraire, la profondeur et la pression interne dans le puits) qui entraînent que la roche autour du puits devient dilatante et par conséquent absorbe le liquide existant (pétrole, eau, etc.). En partant d'un modèle mathématique on discute comment on peut influencer l'état de dilatance de la roche entourant le puits. On donne des exemples numériques pour une roche dure et pour une autre beaucoup moins dure.

\section{Abstract}

In connection with the problem of oil recovery, pressure shafts etc., it is important to determine when after drilling, the rock surrounding the well becomes dilatant and when compressible. In the paper an elastic-viscoplastic constitutive equation for rocks able to describe both compressibility and/or dilatancy of the volume of the rock is formulated. The stress, strain and displacement fields around the oil well is then determined. The creep of rock, i.e. the very slow closing in time of the well and the stress relaxation during creep, are also studied with this model. It is then shown when, i.e. for what primary stresses (generally arbitrary), depth and pressure inside the well, the rock around the well becomes dilatant and therefore will absorb the existing fluids (oil, water, etc.). Starting from the mathematical model given it is then discussed how the rock dilatancy phenomenon around the oil well can possibly be avoided. Numerical examples are given for a hard rock and for a softer one. 


\section{INTRODUCTION}

Dans le présent travail on étudie la déformation des roches autour des puits profonds. L'état de contrainte autour des puits verticaux a été étudié dans le cadre de l'élasticité linéaire par plusieurs auteurs (voir par exemple OBERT et DUVALL (1967), JUMIKIS (1979), DREYER (1973), etc.) en supposant que cet état est analogue à celui qu'on a autour d'un trou pratiqué dans une plaque élastique, c'est-à-dire qu'on a supposé un état de contrainte plane. Le champ de déplacement a été étudié en moindre mesure. Le fluage des roches autour des puits verticaux a été étudié par MASSIER et CRISTESCU (1981) en supposant la déformation plane et en utilisant diverses lois de comportement. Cependant un problème très important pour l'industrie pétrolière n'a pas été considéré: après le forage d'un puits la roche autour du puits est-elle dans un état de dilatance ou de compressibilité? Plus précisément, quand la roche autour du puits devientelle dilatante et quand devient-elle compressible? C'est un problème d'importance évidente pour l'industrie pétrolière, pour certains types de puits verticaux et beaucoup d'autres applications. Ce problème va être considéré dans le présent travail en tenant compte de divers cas possibles de contraintes initiales (primaires) dans la roche et de diverses pressions internes dans le puits. La dilatance et la compressibilité de la roche vont être étudiées pendant la déformation par fluage. En partant du modèle mathématique, c'est-à-dire en connaissant quels sont les paramètres qui ont une influence sur ces phénomènes, on peut envisager comment il est possible d'influencer ces phénomènes. On donne des exemples numériques.

\section{FORMULATION DU PROBLÈME}

Considérons un orifice circulaire profond de ravon a, foré dans un milieu infini. On va utiliser les coordonnées cylindriques $r, \theta, z$ avec l'axe $\mathrm{O} z$ - l'axe du puits - dirigé vers le bas. Après le forage du puits, les parois cylindriques $r=a$ du puits peuvent être libres de contraintes:

$r=a$

quel que soit $z \quad \underline{g}^{T} \underline{n}=\underline{0}$

ou sous une certaine contrainte $\mathrm{p}(\mathrm{t})$ d'un gaz ou d'un liquide qui remplit le trou. Cette contrainte peut varier en temps et peut dépendre de la profondeur $h$ :

$r=a$

quel que soit $z \quad \sigma^{T} \underline{n}=p \underline{n}$.

Ici g est le tenseur des contraintes, l'indice supérieur ' signifie «transposée», $n$ est la normale à la paroi du puits. La contrainte in situ avant le forage, c'est-à-dire l'état primaire de contrainte, va être notée par $\mathrm{g}^{\mathrm{P}}$. Après le forage du puits la contrainte dans la roche, noté $\sigma^{S}$ est nommée contrainte secondaire. Enfin $\mathrm{g}^{R}=$ $\sigma^{s}-Q^{P}$ est la contrainte relative. En ce qui concerne la contrainte primaire, on va d'abord supposer que toutes les composantes primaires horizontales $\sigma_{h}$ sont égales et en général distinctes de la composante verticale $\sigma_{\mathrm{v}}$. On suppose que les deux composantes $\sigma_{\mathrm{h}}$ et $\sigma_{v}$ dépendent de la profondeur $h$ et du site (distribution particulière des contraintes dans le site considéré). Les trois cas possibles $\sigma_{h}>\sigma_{v}, \sigma_{h}=\sigma_{v}$ et $\sigma_{\mathrm{h}}<\sigma_{\mathrm{v}}$ vont être considérés. Ensuite on considère un cas plus général, lorsque $\sigma_{h 1}$ est la composante maximale de la contrainte horizontale, $\sigma_{\mathrm{h} 2}$ la composante minimale et $\sigma_{\mathrm{h} 1}, \sigma_{\mathrm{h} 2}, \sigma_{\mathrm{v}}$ sont composantes principales.

Dans les formules (2.1) et (2.2) sont impliquées les composantes secondaires. En composantes relatives les mêmes conditions deviennent:

$\left.\begin{array}{ll}r=\mathrm{a} & \sigma_{r}^{\mathrm{R}}=\sigma_{\mathrm{r}}^{\mathrm{S}}-\sigma_{\mathrm{r}}^{\mathrm{P}}=\mathrm{p}-\sigma_{\mathrm{h}} \\ \text { quel que soit z } & \sigma_{\mathrm{rz}}^{\mathrm{R}}=\sigma_{\mathrm{r \theta}}^{\mathrm{R}}=0\end{array}\right\}$

A l'infini on a: $\quad \stackrel{\sigma}{\mathrm{R}}^{\mathrm{R}}=0$.

Les contraintes doivent satisfaire l'équation d'équilibre

$$
\frac{\partial \sigma_{r}}{\partial r}+\frac{\sigma_{r}-\sigma_{\theta}}{r}=0
$$

On va supposer comme chez MASSIER et CRISTESCU (1981) que due à la symétrie du problème la seule composante du déplacement est radiale et que le problème dépend d'une seule coordonnée r.

Les composantes de la déformation qui sont impliquées sont:

$$
\varepsilon_{\mathrm{r}}=\frac{\partial \mathrm{u}}{\partial \mathrm{r}}, \quad \varepsilon_{\theta}=\frac{\mathrm{u}}{\mathrm{r}}, \quad \varepsilon_{\mathrm{z}}=0
$$

où u est le déplacement radial. Les composantes (2.6) sont principales. Elles doivent satisfaire la condition de compatibilité

$$
\frac{\partial \varepsilon_{\theta}^{R}}{\partial r}+\frac{\varepsilon_{\theta}^{R}-\varepsilon_{r}^{R}}{r}=0
$$

On suppose que les déplacements et les déformations sont petits.

\section{LOI DE COMPORTEMENT}

Pour étudier la dilatance ou la compressibilité de la roche pendant le fluage on va utiliser la loi de comportement suivante [CRISTESCU $(1984 \mathrm{a}, \mathrm{b})$ ] :

$\dot{\varepsilon}=\left(\frac{1}{3 K}-\frac{1}{2 G}\right) \dot{\sigma} \underset{\sim}{1}+\frac{1}{2 G} \underset{\sim}{\sim}+k$

$\left\langle 1-\frac{W^{\prime}(t)}{H}\right\rangle \frac{\partial H}{\partial \sigma}$

où $\mathrm{G}$ et $\mathrm{K}(\sigma)$ sont les paramètres élastiques. Nous supposerons que $\mathrm{K}$ peut varier très lentement avec la contrainte mais il dépend seulement de la contrainte moyenne:

$$
\sigma=\frac{\sigma_{r}+\sigma_{\theta}+\sigma_{z}}{3}
$$


et que $G$ est constant. Une dépendance possible des paramètres $\mathrm{K}$ et $\mathrm{G}$ par rapport à la contrainte équivalente:

$\hat{\sigma}^{2}=\sigma_{1}^{2}+\sigma_{2}^{2}+\sigma_{3}^{2}-\sigma_{1} \sigma_{2}-\sigma_{2} \sigma_{3}-\sigma_{3} \sigma_{1}$

et par rapport au troisième invariant de la contrainte det $\mathscr{l}=\Delta$, dans le cadre d'un modèle plus général que (3.1), n'est pas connue à présent. $H(\sigma, \bar{\sigma}, \Delta)$ est un potentiel viscoplastique, et

$$
H(\sigma, \bar{\sigma}, \Delta)=W^{l}(t)
$$

est l'équation de la frontière où s'annule la vitesse viscoplastique et

$$
W^{l}(t)=\int_{0}^{t} g(\tau) ; \dot{\sim}^{1}(\tau) d \tau
$$

est le travail irréversible de la contrainte.

Dans les exemples qui suivent on va supposer que $H$ dépend seulement de $\sigma$ et $\sigma$, la dépendance possible par rapport à $\Delta$ est encore inconnue. La notation $\left\langle>\right.$ a le sens $\langle Z\rangle=\frac{1}{2}(Z+|Z|)$. Finalement $k$ $(\sigma, \bar{\sigma}, \Delta, d)$ est un coefficient de "viscosité » quî peut dépendre des invariants de la contrainte et d'un "paramètre de dommage» qui sera défini plus tard.

La loi de comportement (3.1) peut décrire aussi bien la dilatance que la compressibilité de la roche car pour un état particulier de contrainte le signe de la vitesse volumétrique de déformation:

$$
\dot{\varepsilon}_{\mathrm{v}}^{\mathrm{i}}=\mathrm{k}\left\langle 1-\frac{\mathrm{W}^{\prime}(\mathrm{t})}{\mathrm{H}}\right\rangle \frac{\partial \mathrm{H}}{\partial \sigma}
$$

est établi par le signe de la dérivée $\frac{\partial H}{\partial \sigma}$

$\mathrm{Si} \frac{\partial \mathrm{H}}{\partial \sigma}>0$ pendant le fluage une diminution de volume a lieu (compressibilité). Si par contre $\frac{\partial H}{\partial \sigma}<0$ une dilatance se produit. Les états de contraintes qui satisfont l'équation $\frac{\partial \mathrm{H}}{\partial \sigma}=0$ sont par définition sur la frontière compressibilité/dilatance.

On va donner deux exemples. Le premier exemple est basé sur les données expérimentales de BRACE (1965), BRACE, PAULDING et SCHOLZ (1966) et VOLAROVICH et collab. (1974) obtenues pour du granite. Pour cette roche dure le potentiel est:

$$
\begin{aligned}
& H(\sigma, \hat{\sigma}) \equiv\left[\frac{a_{o}}{\left(\sigma-\frac{\bar{\sigma}}{3}\right) \frac{1}{\sigma_{*}}+a_{1}}+a_{2}\right]\left(\frac{\bar{\sigma}}{\sigma_{*}}\right)^{4} \\
& +\left[\frac{\left(\left(\sigma-\frac{\bar{\sigma}}{3}\right) \frac{1}{\sigma_{*}}+b_{o}\right)^{2}}{b_{1}}+b_{2}\right]\left(\frac{\bar{\sigma}}{\sigma_{*}}\right)^{4}
\end{aligned}
$$

$$
\begin{aligned}
& +\left\{\begin{array}{l}
c_{0} \sin \left(\omega \frac{\sigma}{\sigma \cdot}+\varphi\right)+c_{1}, \quad 0 \leqslant \sigma \leqslant \sigma_{0} \\
c_{0}+c_{1} \quad, \quad \sigma_{0} \leqslant \sigma
\end{array}\right. \\
& \text { avec } \mathrm{a}_{\mathrm{o}}=3.833 \cdot 10^{-4} \mathrm{GPa} \text {, } \\
& \mathrm{a}_{1}=9.225 .10^{-3} \\
& \mathrm{a}_{2}=2.622 .10^{-4^{\prime}} \mathrm{GPa} \text {, } \\
& \mathrm{b}_{\mathrm{o}}=6.005 .10^{-3} \text {, } \\
& b_{1}=-969.4893 \mathrm{GPa}^{-1} \\
& \mathrm{~b}_{2}=4.508 .10^{-4} \mathrm{GPa} \text {, } \\
& \mathrm{c}_{\mathrm{o}}=1.16454 .10^{-4} \mathrm{GPa} \text {, } \\
& c_{1}=1.14645 .10^{-4} \mathrm{GPa}, \omega=493.14748^{\circ} \text {, } \\
& \varphi=-79.889307^{\circ}, \\
& \sigma_{*}=1 \mathrm{GPa} \text {, } \\
& \sigma_{o}=0.3445 \mathrm{GPa} \text {. }
\end{aligned}
$$

Les essais hydrostatiques ont suggéré que le paramètre $\mathrm{K}$ est variable et dépend de la contrainte moyenne conformément à la loi empirique:

$K(\sigma)=\left\{\begin{array}{l}K_{0}-K_{1}\left(1-\frac{\sigma}{\sigma_{0}}\right)^{4} \quad \text { si } \quad \sigma \leqslant \sigma_{0} \\ K_{0} \quad \text { si } \quad \sigma \geqslant \sigma_{0}\end{array}\right.$

où $\mathrm{K}_{\mathrm{o}}=59 \mathrm{GPa}$ et $\mathrm{K}_{1}=48 \mathrm{GPa}$. Si cependant $\sigma$ est presque constant on va supposer que les pentes

$$
\frac{1}{3 G}+\frac{1}{9 K} \quad, \quad-\frac{1}{6 G}+\frac{1}{9 K}
$$

des courbes caractéristiques peuvent avoir des valeurs presque constantes, quî dépendent néanmoins de la profondeur. Des valeurs moyennes constantes qui soient raisonnables pour des profondeurs pas trop grandes sont $\mathrm{G}=32.3 \mathrm{GPa}$ et $\mathrm{K}=51.3 \mathrm{GPa}$.

Dans l'exemple qui suit on suppose que $\mathrm{H}$ et $\mathrm{k}$ sont des fonctions qui dépendent seulement des invariants $\sigma$ et $\sigma$. Avec (3.7) et les valeurs des divers paramètres donnés, la loi de comportement est entièrement déterminée à l'exception de la valeur de $\mathrm{k}$ qui va être discutée plus loin. Conformément à cette loi de comportement, utilisée seulement pour des contraintes positives, dans le domaine $D_{c}$ (fig. 1) la roche est compressible, mais dilatante dans le domaine $\mathrm{D}_{\mathrm{d}}$. Une ligne frontière sépare les deux domaines et sa forme est déterminée très précisément par l'équation:

$$
\frac{\partial \mathrm{H}}{\partial \sigma}=0
$$

Le long de la ligne hydrostatique $\bar{\sigma}=0$, pour $\sigma \geqslant \sigma_{0}$, le comportement de la roche est linéaire élastique. Pour toutes les autres contraintes possibles (c'est-à-dire qui ne produisent pas une rupture) le comportement de la roche est viscoplastique avec une limite de plasticité nulle. La roche est viscoplastique même pendant les chargements hydrostatiques si $\sigma<\sigma_{0}$. 
Le deuxième exemple est pour une roche moins dure. C'est un andésite (de Suior-Roumanie). Pour cette roche le potentiel viscoplastique est:

$$
\begin{aligned}
& H(\sigma, \bar{\sigma}) \equiv \frac{\frac{a_{o}}{\left(\sigma-\frac{\bar{\sigma}}{3}\right) \frac{1}{\sigma_{*}}+a_{1}}\left(\frac{\bar{\sigma}}{\sigma_{*}}\right)^{4}}{+\frac{\left[\frac{1}{\sigma *}\left(\sigma-\frac{\bar{\sigma}}{3}\right)-b_{0}\right]^{2} \frac{\bar{\sigma}}{\sigma_{*}}+\frac{1}{2}\left(\frac{1}{K_{0}}-\frac{1}{K}\right) \sigma^{2}+c \sigma}{+b_{1}}}
\end{aligned}
$$

avec $a_{0}=3.2855 .10^{-3} \mathrm{GPa}$,

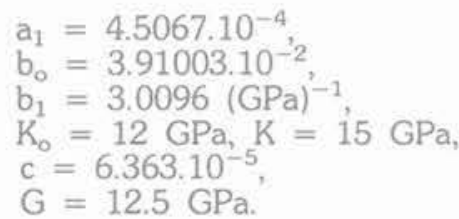

Ce modèle a été obtenu pour des pressions moyennes assez petites et en conséquence peut être utilisé pour des profondeurs qui ne dépassent pas $1 \mathrm{~km}$ environ. En ce qui concerne le dommage et la rupture possible de la roche, on va considérer deux cas. Si $\sigma<0$ et si le chargement est assez brusque on va supposer que le comportement de la roche est très proche d'un comportement linéaire élastique et la rupture se produit quand la composante principale minimale de la contrainte atteint une certaine valeur limite qui soit une constante caractéristique de la roche. Si $\sigma>0$ la rupture est possible par un processus de dommage produit par la dilatance. On introduit un paramètre de dommage par:

$$
d(t)=W_{v \max }^{i}-W_{v}^{i}(t)
$$

où

$$
W_{v \max }^{1}(T)=\int_{0}^{T} g(t): \dot{\varepsilon}_{d}^{I}(t) d t
$$

et $W_{v \max }^{I}$ est la valeur maximale positive de $W_{v}^{l}$ dans l'intervalle $t \in[0, T]$. On va supposer que le coefficient de viscosité $k$ dépend aussi de $d$ et que pour une certaine valeur critique $\mathrm{d}_{\mathrm{cr}}$, quand $\mathrm{d} \rightarrow \mathrm{d}_{\mathrm{cr}}$ on a $\mathrm{k} \rightarrow \infty$. C'est-à-dire qu'on suppose ici un critérium énergétique pour déterminer la rupture, par l'influence du paramètre de dommage sur le coefficient de viscosité.

\section{L'ÉTAT SECONDAIRE INITIAL}

Pour décrire d'une façon pratique le fluage de la roche qui se trouve autour du puits, deux intervalles de temps vont être considérés dans le problème. Le premier intervalle de temps $t \in\left[0, t_{0}\right]$ correspond au temps nécessaire pour forer le puits (ou une partie du puits). Le deuxième intervalle $t \in\left[t_{0}, t_{1}\right]$ est l'intervalle de temps pendant lequel le puits est en exploitation. On va supposer ici que le premier intervalle de temps est beaucoup plus court que le deuxième. En conséquence la déformation que la roche subit pendant le premier intervalle peut être considérée comme *instantanée» en comparaison de la déformation qui a lieu dans le deuxième intervalle de temps. Conformément au modèle (3.1) la réponse «instantanée est une réponse élastique et le fluage qui a lieu dans le deuxième intervalle de temps sera décrit par la loi de comportement (3.1) tout entière. Notons par l'indice supérieur ${ }^{R_{0}}$ les composantes relatives au moment $t_{0}$, obtenues immédiatement après le forage et par l'indice supérieur ${ }^{S_{0}}$ les composantes secondaires initiales. On a [voir par exemple MASSIER et CRISTESCU (1981)]:

$$
\begin{aligned}
& \sigma_{r}^{R_{0}}=\sigma_{r}^{S_{0}}-\sigma_{r}^{P}=\left(p-\sigma_{h}\right) \frac{a^{2}}{r^{2}} \\
& \sigma_{\theta}^{R_{0}}=-\left(p-\sigma_{h}\right) \frac{a^{2}}{r^{2}}, \sigma_{z}^{R_{0}}=0 \\
& \varepsilon_{r}^{R_{0}}=\varepsilon_{r}^{S_{0}}-\varepsilon_{r}^{P}=\frac{p-\sigma_{h}}{2 G} \frac{a^{2}}{r^{2}} \\
& \varepsilon_{\theta}^{R_{0}}=-\frac{p-\sigma_{h}}{2 G} \frac{a^{2}}{r^{2}}, \varepsilon_{z}^{R_{0}}=0 \\
& u^{R_{0}}=-\frac{p-\sigma_{h}}{2 G} \frac{a^{2}}{r}, W^{R_{0}}=0 .
\end{aligned}
$$

Parce que pour les états primaires et secondaires on a pour la contrainte moyenne et la contrainte équivalente:

$$
\begin{aligned}
& \sigma^{S_{0}}=\sigma^{p}=\frac{2 \sigma_{h}+\sigma_{v}}{3} \\
& \left(\sigma^{p}\right)^{2}=\left(\sigma_{h}-\sigma_{v}\right)^{2} \\
& \left(\sigma^{S_{0}}\right)^{2}=3\left(p-\sigma_{h}\right)^{2} \frac{a^{4}}{r^{4}}+\left(\sigma_{v}-\sigma_{h}\right)^{2}
\end{aligned}
$$

et parce que $\dot{\sigma}^{s_{0}} \geqslant \bar{\sigma}^{p}$ par le forage on a produit un "chargement». Pour donner un exemple, supposons que la distribution des contraintes primaires soit donnée par des formules du type:

$$
\sigma_{v}=0,027 \mathrm{~h}, \sigma_{\mathrm{h}}=5.4+0,0216 \mathrm{~h}
$$

suggérées par les travaux de HAIMSON (1978) et BROWN et HOEK (1978), où la profondeur h est donnée en mètres et les contraintes en MPa. N'importe quelle autre distribution des contraintes primaires peut être considérée d'une façon semblable. Sur la figure 1 pour le granite, les états primaires à des profondeurs diverses $(0,5 \mathrm{~km}, 1 \mathrm{~km}, 5 \mathrm{~km}, 9 \mathrm{~km}$ et $11 \mathrm{~km})$ sont marqués par des cercles. Les états secondondaires correspondants sont marqués par des carrés. On constate que si la profondeur augmente, les états secondaires s'éloignent beaucoup des états primaires. Il est donc possible qu'à une certaine profondeur l'état secondaire initial soit placé dans le domaine $\mathrm{D}_{\mathrm{d}}$ où a lieu une dilatance. On peut remarquer que à $\mathrm{h}=1 \mathrm{~km}$ l'état primaire est situé sur la droite hydrostatique. Tous les états primaires et secondaires 
montrés sur cette figure ont été calculés pour $\mathrm{p}=0$ à $r=a$. L'exemple numérique qui a été donné ici veut illustrer seulement l'idée que l'état secondaire peut être situé dans le domaine dilatant très loin de l'état primaire. Une estimation plus exacte du passage dans le domaine dilatant par un forage nécessite une détermination assez précise de l'état primaire de contraintes dans le site considéré. La loi (4.3) suggère seulement des valeurs moyennes et en tout cas elle ne peut être utilisée que jusqu'à $3 \mathrm{~km}$ de profondeur.

\section{FLUAGE DES ROCHES AUTOUR DES PUITS VERTICAUX ET DES FORAGES PÉTROLIERS}

Le fluage de la roche autour du puits est décrit dans l'intervalle très long de temps $t \in\left[t_{0}, t_{1}\right]$ par la loi de comportement (3.1).

On va supposer que, in situ, les états primaires de contraintes sont des états d'équilibre, c'est-à-dire que $\dot{\varepsilon}=\mathrm{g}=\mathrm{l}$. Alors de $(3.1)$ résulte $\mathrm{H}\left(\sigma^{\mathrm{P}}, \tilde{\sigma}^{\mathrm{P}}, \Delta^{\mathrm{P}}\right)=$ $W^{I P}$. Ici $W^{\mathbb{I P}}$ est un nombre qui est tout simplement la valeur de $\mathrm{H}$ pour l'état primaire et qui a dans le modèle un rôle conventionnel. Après le forage instantané qui a changé l'état de contrainte primaire $\sigma^{P}$ en un état secondaire instantané $\sigma^{S_{0}}=\sigma^{p}+\sigma^{R_{0}}$ et qui n'a pas produit une variation de $W^{l}(t)$ (parce que $W^{\prime}(t)$ ne peut varier qu'en fonction du temps), suit une période très longue de temps dans laquelle a lieu le fluage. Dans ce qui suit on va présenter deux solutions. On va d'abord donner une solution simplifiée, moins rigoureuse mais très simple, qu'on peut obtenir en supposant que pendant le fluage les contraintes restent constantes et égales à o $\sigma^{5_{0}}$. On donnera ensuite une solution générale numérique.

Si dans l'intervalle $t \in\left\{t_{0}, t_{1}\right\}$ les contraintes $\sigma^{S_{0}}$ sont constantes, alors le travail irréversible varie conformément à la loi:

$$
\begin{aligned}
& 1-\frac{W^{\prime}(t)}{H(\sigma, \bar{\sigma}, \Delta)}=\left(1-\frac{W^{\mathbb{I P}}}{H(\sigma, \bar{\sigma}, \Delta)}\right) \\
& \exp \left[\frac{k}{H} \cdot \frac{\partial H}{\partial \sigma} \cdot \stackrel{\sim}{\sim}\left(t_{0}-t\right)\right]
\end{aligned}
$$

qu'on obtient de (3.1). Dans (5.1) $q=\alpha^{S_{0}}$ est une contrainte constante. En introduisant (5.1) dans (3.1) on obtient pour la variation de la déformation en fonction du temps:

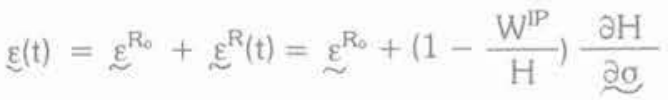

$$
\begin{aligned}
& \frac{1}{\mathrm{H}} \frac{\partial \mathrm{H}}{\partial \sigma} \cdot \stackrel{\sigma}{\sim}\{1-\exp \\
& \left.\left[\frac{k}{H} \frac{\partial H}{\partial \sigma} \quad \sigma\left(t_{0}-t\right)\right]\right\}
\end{aligned}
$$

avec les conditions initiales à $\mathrm{t}=\mathrm{t}_{\mathrm{o}}$

$$
\begin{aligned}
& \mathrm{t}=0: \quad \mathrm{\varepsilon}_{1}^{\mathrm{t}}=0
\end{aligned}
$$

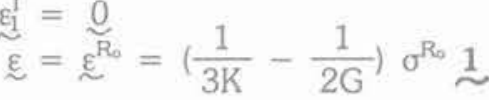

$$
\begin{aligned}
& +\frac{1}{2 G} \stackrel{\sigma^{R_{0}}}{\sim}
\end{aligned}
$$

On doit observer que généralement les déformations (5.2) ne satisfont pas l'équation de compatibilité (2.7) et que grâce à l'hypothèse de la déformation plane, la condition $\varepsilon_{2}=0$ impose une restriction pour la variation de $\sigma_{Z}$ :

$$
\frac{1}{3}\left(\frac{1}{3 K}+\frac{1}{G}\right) \dot{\sigma}_{z}^{R}=-k\left(1-\frac{W^{l}}{H}\right) \frac{\partial H}{\partial \sigma_{z}}
$$

Pour obtenir une solution générale par des moyens numériques; on doit intégrer le système d'équations (2.5). (2.7) et (5.1) avec cing fonctions inconnues $\varepsilon_{r}^{\mathrm{R}}$ $\varepsilon_{\theta}^{R}, \sigma_{r}^{s}, \sigma_{\theta}^{S}$ et $\sigma_{z}^{s}$ et deux variables indépendants $r$ et $t$. Les conditions initiales sont:

$$
\begin{aligned}
t=t_{0}, r \geqslant a: & \\
\sigma_{r}^{S o} & =\left(p-\sigma_{h}\right) \frac{a^{2}}{r^{2}}+\sigma_{h} \\
\sigma_{\theta}^{S o} & =-\left(p-\sigma_{h}\right) \frac{a^{2}}{r^{2}}+\sigma_{h} \\
\sigma_{z}^{S o} & =\sigma_{v}, \underset{\sim}{\varepsilon}={\underset{\varepsilon}{\sim}}^{R_{0}}
\end{aligned}
$$

où les déformations $\varepsilon^{R_{0}}$ sont fournies par (4.1). Les conditions aux limites sont:

$$
\begin{aligned}
r=a, t \geqslant 0: & \sigma_{r}=p \\
r \rightarrow \infty, t \geqslant 0: & \\
\sigma_{r}^{S} & =\sigma_{\theta}^{S}=\sigma_{h}, \sigma_{z}=\sigma_{v} \\
\varepsilon_{r}^{R} & =\varepsilon_{\theta}^{R}=\varepsilon_{z}^{R}=0 .
\end{aligned}
$$

Le système (2.5), (2.7) et (3.1) est écrit en différences finies sur un réseau de droites $r=$ const. tracées à des intervalles $\Delta r$ croissants avec $r$ et des intervalles $\Delta t$ croissants avec t. Par une méthode de balayage on peut raccorder les conditions aux limites (5.6) et (5.7).

Les calculs ont été faits pour l'andésite à potentiel (3.9) avec $\mathrm{k}=5,69 \cdot 10^{-} \mathrm{T}^{-1}$ et une profondeur $\mathrm{h}=$ $500 \mathrm{~m}$, l'êtat primaire étant donné par (4.3). On a poussé les calculs jusqu'à $t-t_{0}=166$ jours, lorsque conformément à ce modèle pour la valeur particulière de $k$ utilisée, le fluage de la roche se stabilise autour du puits. Pour une roche donnée et pour une profondeur donnée, une valeur plus exacte de $\mathrm{k}$ peut être établie seulement par mesures faites in situ. C'est seulement alors que le temps de stabilisation peut être établi avec une certaine précision.

Les résultats des calculs numériques ont été comparés avec la solution simplifiée (5.2). Les conclusions sont les suivantes:

a. Les contraintes $\sigma_{\mathrm{r}} \quad \sigma_{\theta}$ et $\sigma_{\mathrm{z}}$ varient pendant la période du fluage. La variation de $\sigma_{r}$ est très petite et presque négligeable mais la variation de $\sigma_{\theta}$ est signi- 


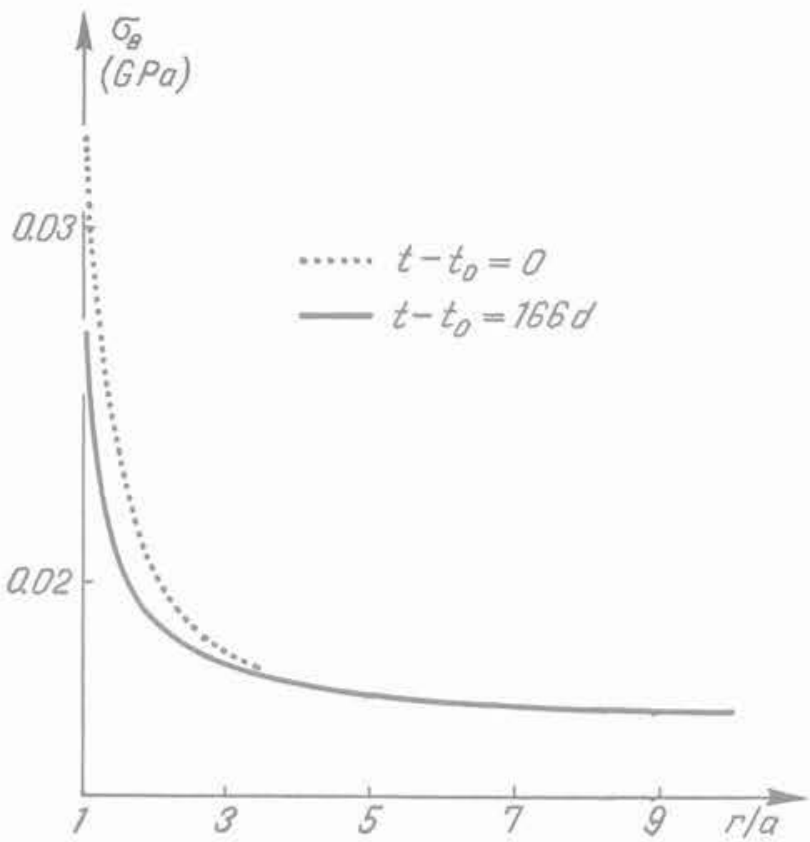

Fig. 2. - Distribution de la contrainte $\sigma_{\theta}$ en fonction de la distance après la déformation élastique initiale et à la fin de la période de fluage.

ficative. Cette variation est donnée dans la figure 2 où par ligne pointillée sont marquées les contraintes fournies par $\sigma_{\theta}^{S_{0}}$ et par ligne continue la contrainte $\sigma_{\theta}$ obtenue après 166 jours avec la méthode numérique. Notons que pendant le fluage la contrainte $\sigma_{\theta}$ subit une relaxation qui diminue sa valeur (qui était $\sigma_{\theta} \mathrm{S}_{0}$ au moment $t=t_{0}$ ) en l'approchant de la valeur primaire $\sigma_{\mathrm{h}}$. La ligne continue dans cette figure montre les valeurs finales de $\sigma_{\theta}$. Pour faire une comparaison dans la figure 3 est donnée la variation de $\sigma_{r}$. Les deux solutions données coïncident à $r=a$ et à grandes distances et diffèrent très peu à des distances intermédiaires. En ce qui concerne $\sigma_{z}$, cette composante est presque constante: une très faible croissance est

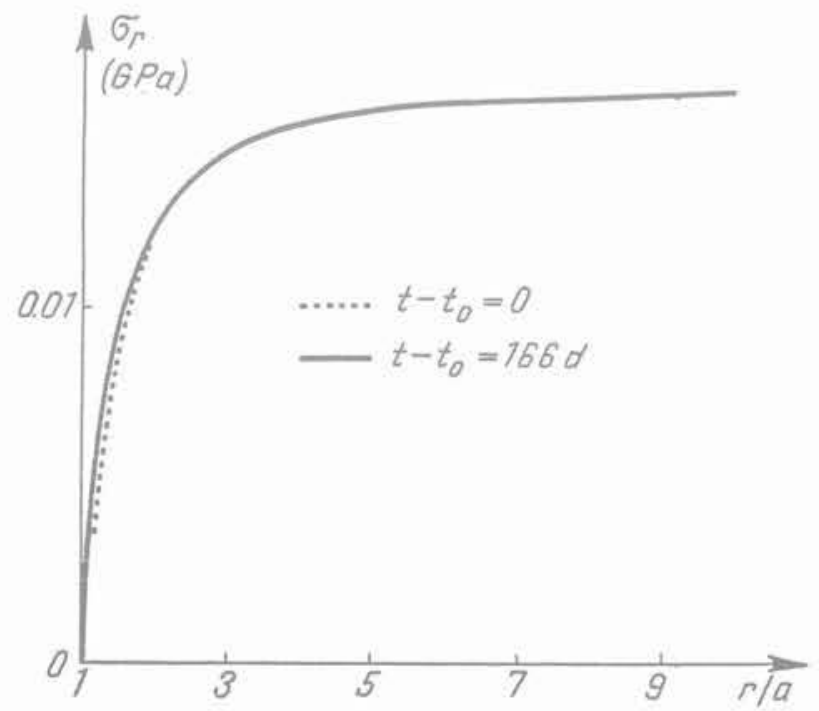

Fig. 3. - Distribution de la contrainte $\sigma_{r}$ en fonction de la distance après la déformation élastique initiale et à la fin de la période de fluage. obtenue au voisinage immédiat de $\mathrm{r}=\mathrm{a}$ (pour $\mathrm{r}<$ 1.2 a). Donc pendant le fluage les contraintes $\sigma_{r}$ et $\sigma_{\theta}$ subissent une certaine relaxation qui les approche de la valeur primaire $\sigma_{\mathrm{h}}$

b. En ce qui concerne les déformations, elles subissent une croissance en valeur absolue, mais cette croissance est beaucoup plus petite que celle obtenue avec la solution simplifiée (5.2). Pour donner un exemple, est montrée la distribution du déplacement radial autour de l'orifice dans la figure 4. La ligne pointillée correspond au déplacement élastique initial (formule $\left.(4.1)_{7}\right)$; la ligne continue correspond à la solution simplifiée (5.2) et $u=r \varepsilon_{\theta}$; la ligne interrompue correspond â la solution numérique.

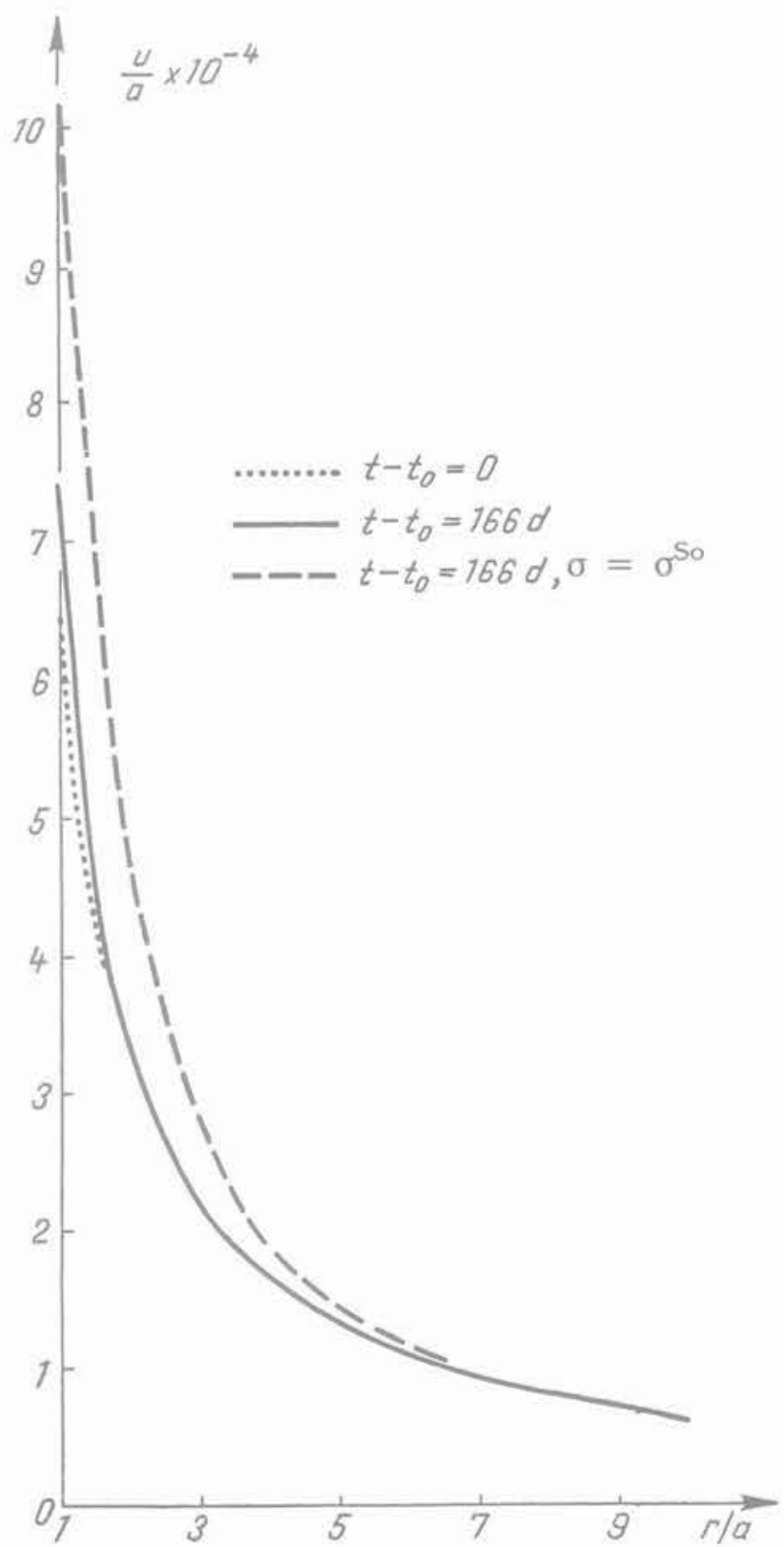

Fig. 4. - Déplacement radial en fonction de la distance: déplacement élastique initial (ligne pointillée), déplacement après fluage (ligne continue) et déplacement obtenu avec la solution simplifiée (ligne interrompue). 
La conclusion est que les déplacements fournis par la solution simplifiée sont plus grands que ceux obtenus plus exactement avec la méthode numérique. Donc la solution simplifiée peut nous donner une première information qui est un majorant de la solution exacte.

\section{LA DILATANCE DE LA ROCHE AUTOUR DU PUITS}

La détermination assez précise de la loi de comportement élasto-viscoplastique capable de décrire la dilatance autant que la compressibilité de la roche conjointement à la connaissance assez exacte de la distribution de l'état primaire des contraintes, peuvent avoir une grande importance pour un forage correct du puits.

Généralement quand on fait un forage à une petite profondeur dans une roche dure, la roche autour du puits se déforme, produisant une compressibilité volumique. Pendant cette compressibilité le fluide existant dans les pores va être évacué. Cependant aux profondeurs plus grandes la roche autour du puits peut devenir dilatante parce que le point représentant l'état secondaire de contraintes peut être situé dans le domaine de dilatance (fig. 1). Dans ce dernier cas la

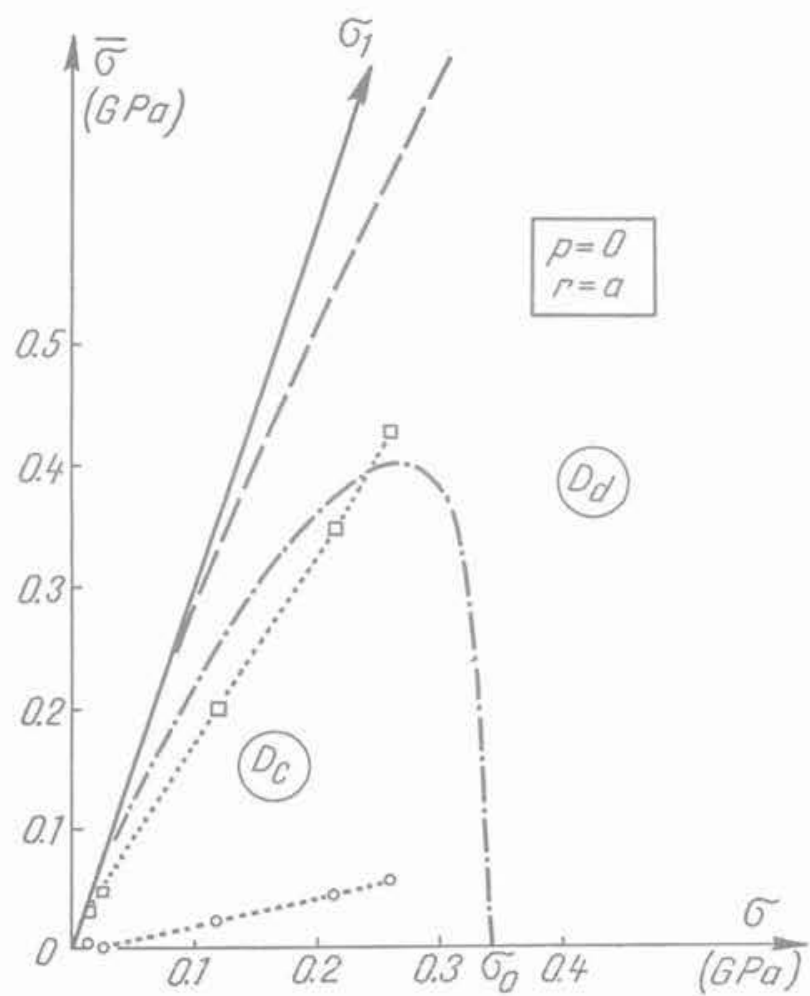

Fig. 1. - Diagramme des contraintes montrant les contraintes primaires (cercles), secondaires (carrés), le domaine de dilatance $D_{d}$, le domaine de compressibilité $D_{c}$ la frontière compressibilité/dilatance (ligne frontière) et la surface de rupture (ligne interrompue). roche va absorber le fluide qui existe dans le puits. La profondeur où cela peut se produire dépend des propriétés mécaniques des roches qu'on trouve le long du puits et des contraintes primaires. En utilisant le modèle mathématique donné plus haut on peut déterminer exactement cette profondeur. Sur la figure 5 la surface autour du puits qui est la frontière entre les régions où la roche est compressible (région $\mathrm{D}_{\mathrm{c}}$ ) et où elle est dilatante (région $D_{d}$ ) est figurée par $A B$.

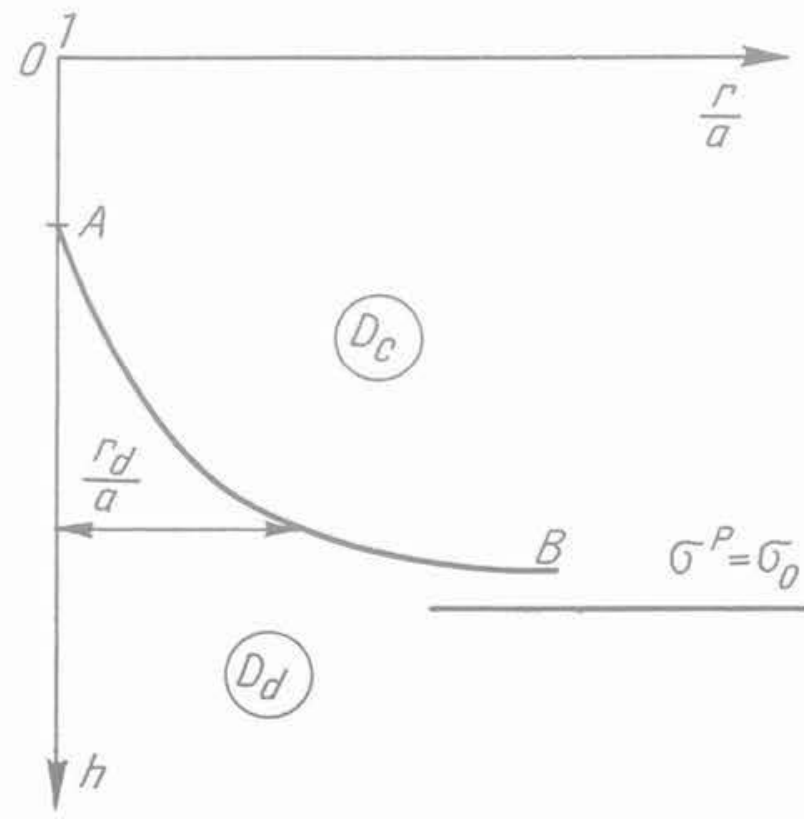

Fig. 5. - Augmentation du rayon de la frontière compressibilité/dilatation avec la profondeur.

Le point $A$ est la profondeur minimale où la roche autour du puits devient dilatante. Quand la profondeur sagrandit, le rayon $\mathrm{r}_{\mathrm{d}} / \mathrm{a}$ de la frontière compressibilitél dilatante s'agrandit. Finalement quand la profondeur est encore plus grande et $\sigma^{p}$ est proche de la valeur $\sigma_{0}$ alors $r_{d} \rightarrow \infty$. Du point de vue pratique une déformation sensible de la roche autour du puits n'a lieu que sur une distance de quelques rayons de puits. Comme exemple numérique pour le granite le point $A$ est situé à une profondeur très grande, d'environ $10 \mathrm{~km}$ et l'asymptote $\sigma^{P}=\sigma_{0}$ est située environ à $14,5 \mathrm{~km}$ de profondeur. Pour l'andésite à toute profondeur la roche autour du puits devient dilatante. Généralement, pour n'importe quelle autre roche et pour une autre distribution des contraintes primaires (déterminées localement) la frontière $A B$ peut être déterminée avec précision, si la loi de comportement est connue.

On peut envisager des méthodes pour maintenir la roche autour du puits dans un état compressible même sous la frontière $A B$, en trouvant des procédés technologiques pour changer les valeurs de quelques paramètres qu'influencent $\bar{\sigma}^{-s_{0}}$ et en conséquence pour maintenir l'état secondaire de contraintes dans le domaine compressible. Ces paramètres sont $\sigma_{\mathrm{h}}, \sigma_{\mathrm{v}}$ tandis que l'expression de $\sigma^{\text {s。 }}$ peut nous suggérer le procédé à suivre. En général on doit changer ces 
paramètres pour réduire la valeur de $\bar{\sigma}^{-s_{0}}$ et en conséquence celle de $r_{\mathrm{d}}$. Si le point qui représente l'état secondaire initial est dans le domaine dilatant et loin de la frontière compressibilité/dilatance, la roche restera de toutes les façons dilatante, parce qu'en changeant les paramètres mentionnés plus haut il ne sera pas possible de changer d'une façon significative cette frontière.

On va donner maintenant un autre exemple pour une roche moins dure, l'andésite, dont le modèle est (3.1), (3.9). Dans ce cas il y a plusieurs situations possibles. Pour en donner un exemple considérons la figure 6 (voir aussi CRISTESCU (1984c, 1985). Dans cette figure, pour l'andésite, sont représentés dans un plan $\sigma, \bar{\sigma}$, les deux domaines $D_{c}$ et $D_{d}$ ainsi que la frontière qui les sépare. $\mathrm{Si}$ la distribution de contraintes primaires est toujours du type (4.3), alors si on n'a pas de pression à l'intérieur du puits $(\mathrm{p}=0)$ l'état de contrainte sur la circonférence $r=a$ aux profondeurs $\mathrm{h}=100 \mathrm{~m}, 300 \mathrm{~m}$ et $500 \mathrm{~m}$ est marqué par des petits cercles.

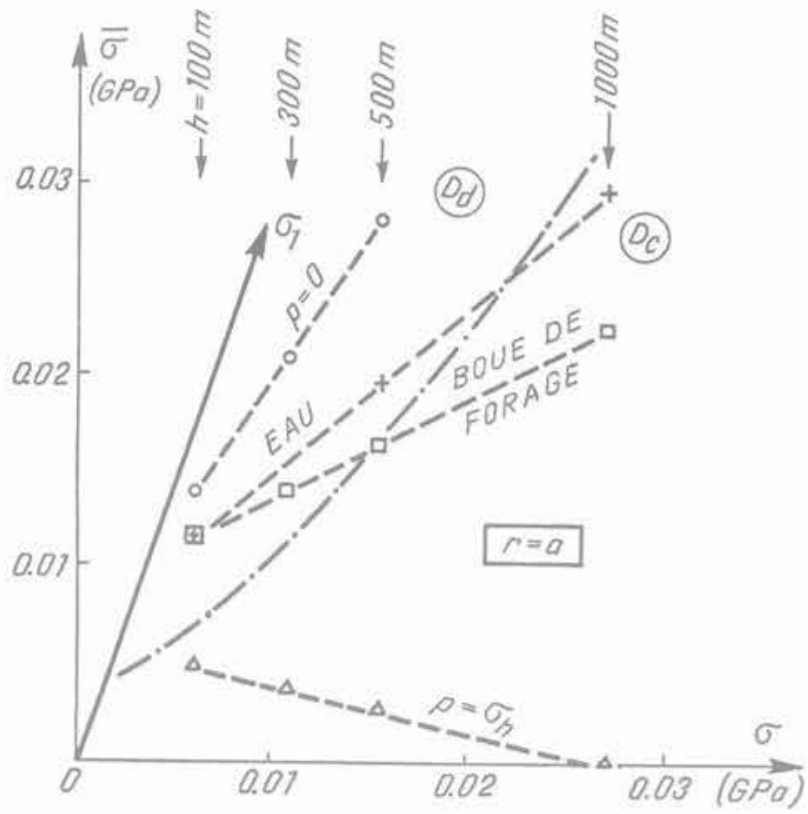

Fig. 6. - Etat des contraintes autour du puits pour: puits vides (cercles), puits rempli d'eau (croix), puits rempli de boue (carrés) et états primaires de contraintes (triangles).

En conséquence à toutes les profondeurs (en partant mêrne de $\mathrm{h}=0 \mathrm{~m}$ ) la roche autour du puits devient dilatante. Les choses peuvent changer si sur la paroi $\mathrm{r}=\mathrm{a}$ on applique une certaine pression $\mathrm{p} \neq 0$. Par exemple si le puits est rempli d'eau, alors l'état de contrainte pour $r=a$ et aux diverses profondeurs est marqué par de petites croix. Donc aux petites profondeurs la roche est aussi dilatante tandis qu'aux profondeurs plus grandes (d'environ 700 ou $800 \mathrm{~m}$ ) la roche devient compressible autour du puits. Si le puits est rempli de boue de forage qui fournit une pression de $14 \mathrm{kPa}$ par mètre de profondeur (COATES (1981) p. 3.6) alors les états de contrainte aux profondeurs $100 \mathrm{~m}, 300 \mathrm{~m}, 500 \mathrm{~m}$ et $1000 \mathrm{~m}$ sont marqués par de petits carrés. Dans ce cas-ci, sous $500 \mathrm{~m}$ la roche est compressible autour du puits. Par comparaison, les états de contrainte pour le cas limite $\mathrm{p}=\sigma_{\mathrm{h}}$ sont représentés aux mêmes profondeurs par des triangles, c'est-à-dire les contraintes primaires conformément aux formules (4.3).

Dans l'exemple donné plus haut, les domaines dilatants ou compressibles autour du puits, pour $r=a$, sont seulement les domaines initiaux. Pourtant comme on l'a montré au paragraphe antérieur, pendant le fluage a lieu une relaxation de l'état de contrainte. Cette relaxation produit une décroissance lente assez marquée de $\bar{\sigma}$ et aussi une faible décroissance de $\sigma$. En conséquence on peut trouver des régions autour du puits qui pendant le fluage commencent par être dilatantes et finissent par être compressibles. Pour en donner un exemple revenons à la solution numérique donnée plus haut pour l'andésite à la profondeur de $500 \mathrm{~m}$. La décroissance de contraintes est représentée sur la figure 7 . Ici dans un plan $\sigma, \sigma$ sont représentés le domaine $\mathrm{D}_{\mathrm{d}}$ des contraintes qui produisent la dilatance de l'andésite et le domaine $D_{c}$ où la roche est compressible. La ligne frontière est la frontière compressibilité/dilatance dont l'équation est $\partial \mathrm{H} / \partial \sigma=0$ peut être obtenue facilement de (3.9). L'état primaire de contraintes est marqué par le point P. L'état secondaire $\sigma^{\mathrm{S}_{0}}$ est marqué par 0 pour trois distances $r=a, r=1.2 a$ et $r=2 a$. Ces trois points sont situés sur le même plan octaédrique que $\mathrm{P}$. Pendant le fluage a lieu une relaxation de la contrainte, donc ơ et

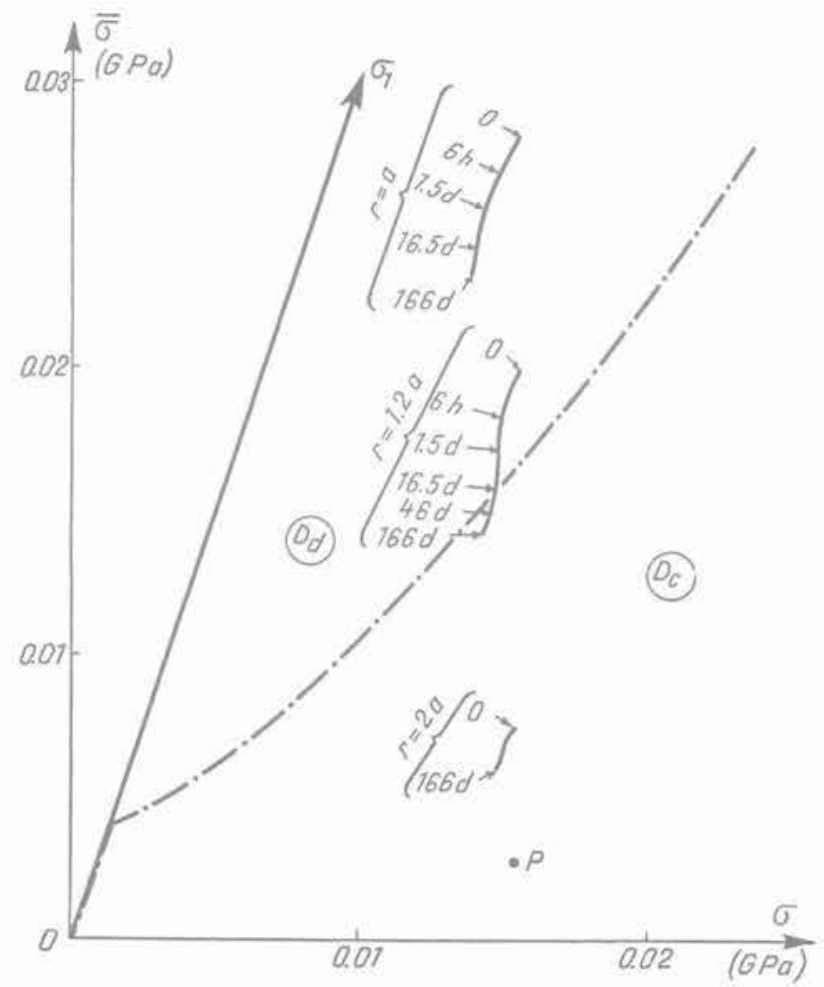

Fig. 7. - Relaxation de la contrainte pendant le fluage produisant seulement la compressibilité (à $r=2$ a) ou seulement la dilatance (à $r=a$ ) ou d'abord la dilatance et ensuite la compressibilité (à $r=1.2 \mathrm{a}$ ). 
o diminuent. Cette décroissance est indiquée sur la figure par les trois valeurs de $r$. Le point final correspond à 166 jours après le commencement du fluage. A remarquer qu'à des distances très voisines du puits la roche reste dilatante pendant toute la période du fluage. A des distances relativement grandes (par exemple $r>1.5 \mathrm{a}$ ) la roche est compressible pendant la période du fluage. Enfin à distance intermédiaire pendant le fluage, la roche commence par être dilatante et finit par être compressible. Cela est illustré sur la figure pour $r=1.2 \mathrm{a}$.

Une conclusion intéressante est obtenue en ce qui concerne la déformation irréversible de volume $\tilde{\varepsilon}_{v}^{I}=\varepsilon_{r}^{I}+\varepsilon_{\theta}^{\prime}+\varepsilon_{2}^{I}$ pendant le fluage, pour diverses distances radiales $r$. Notons que $\varepsilon_{2}=0$ et donc $\varepsilon_{2}^{1}=$ - $\varepsilon_{z}^{\mathrm{E}}$ avec la déformation élastique $\varepsilon_{2}^{\mathrm{E}}$ calculée avec la méthode numérique mentionnée plus haut. Pour en donner un exemple sur la figure 8 on montre la déformation volumique, irréversible après 2,5 jours et 166 jours, en fonction de la distance. Donc au voisinage immédiat du puits la déformation volumique irréversible décroit continuellement, pourtant aux grandes distances elle croît continuellement et finalement aux distances intermédiaires la déformation irréversible de volume commence par décroître au commencement de la période du fluage et finit par croître à la fin de cette période. Globalement une tranche $z=$ const. d'épaisseur unitaire va subir un processus de compressibilité, c'est-à-dire une portion plus grande de volume de cette tranche est dans un état de compressibilité par rapport à la portion qui est dans un état de dilatance.

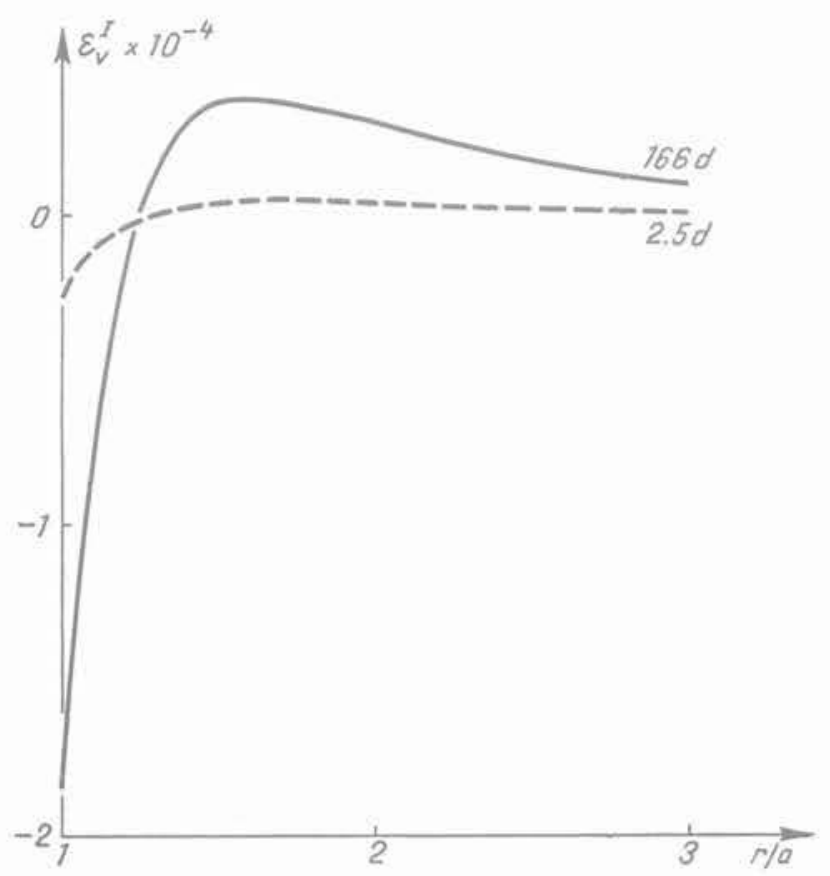

Fig. 8. - Déformation volumique irréversible à deux intervalles de temps montrant la dilatance près de l'orifice et la compressibilité à des distances plus grandes de l'orifice.

\section{UN ÉTAT PRIMAIRE DE CONTRAINTE PLUS GÉNÉRAL}

Dans les paragraphes antérieurs on a supposé qu'à toutes les profondeurs toutes les composantes primaires horizontales sont égales. En réalité les données expérimentales nous montrent que dans diverses directions horizontales et dépendant de la profondeur, les contraintes normales primaires peuvent prendre différentes valeurs. Par exemple LINDNER et HALPERN (1978) ont synthétisé les résultats obtenus par divers auteurs qui ont fait des mesures en Amérique du Nord; ces résultats expérimentaux montrent que, en fonction du lieu et de la profondeur, dans le plan horizontal il y a une direction où $\sigma_{\mathrm{h}}$ est maximale et une direction orthogonale où $\sigma_{\mathrm{h}}$ est minimale. Des conclusions semblables ont été obtenues par SAXENA et coll. (1979), ABOU-SAYET et coll. (1978) et d'autres auteurs.

Supposons maintenant qu'on ait une valeur maximale $\sigma_{h 1}$ et une valeur minimale $\sigma_{h 2}$ de $\sigma_{h}$ et que $\sigma_{h 1}, \sigma_{h 2}$ et $\sigma_{y}$ soient des contraintes principales. Cette dernière supposition n'est pas toujours satisfaite parce que les directions principales des contraintes primaires ont généralement une orientation arbitraire. Bien sûr, on va supposer que les composantes primaires sont connues et généralement distinctes, avec $\sigma_{h 1}>\sigma_{h 2}$.

L'état de contraintes autour d'un puits vertical peut être obtenu dans ce cas par des formules similaires à celles utilisées pour les tunnels horizontaux (voir par exemple MASSIER et CRISTESCU (1984)). Considérons un plan horizontal de profondeur arbitraire. Quand pour $z=$ const. on fait $r \rightarrow \infty$ on doit avoir:

$\left.\sigma_{x x}^{s}\right|_{\infty}=\sigma_{h 1},\left.\sigma_{y y}^{s}\right|_{\infty}=\sigma_{h 2},\left.\sigma_{z z}^{s}\right|_{\infty}=\sigma_{v}$

En coordonnées cylindriques les composantes primaires sont:

$$
\begin{aligned}
\left.\sigma_{\pi}^{\mathrm{S}}\right|_{\infty} & =\sigma_{\pi}^{\mathrm{P}}=\frac{1}{2}\left(\sigma_{\mathrm{h} 1}+\sigma_{\mathrm{h} 2}\right) \\
& +\frac{1}{2}\left(\sigma_{\mathrm{h} 1}-\sigma_{\mathrm{h} 2}\right) \cos 2 \theta \\
\left.\sigma_{\theta \theta}^{\mathrm{S}}\right|_{\infty} & =\sigma_{\theta \theta}^{\mathrm{P}}=\frac{1}{2}\left(\sigma_{\mathrm{h} 1}+\sigma_{\mathrm{h} 2}\right) \\
& -\frac{1}{2}\left(\sigma_{\mathrm{h} 1}-\sigma_{\mathrm{h} 2}\right) \cos 2 \theta \\
\left.\sigma_{r \theta}^{\mathrm{S}}\right|_{\infty} & =\sigma_{\mathrm{r} \theta}^{\mathrm{P}}=-\frac{1}{2}\left(\sigma_{\mathrm{h} 1}-\sigma_{\mathrm{h} 2}\right) \sin 2 \theta \\
\left.\sigma_{z 2}^{\mathrm{S}}\right|_{\infty} & =\sigma_{z z}^{\mathrm{P}}=\sigma_{\mathrm{v}}
\end{aligned}
$$

Les composantes relatives peuvent être obtenues de:

$$
\begin{aligned}
\sigma_{r}^{R}= & p \frac{a^{2}}{r^{2}}-\frac{\sigma_{h 1}+\sigma_{h 2}}{2} \frac{a^{2}}{r^{2}} \\
& -\frac{\sigma_{h 1}-\sigma_{h 2}}{2}\left(\frac{4 a^{2}}{r^{2}}-\frac{3 a^{4}}{r^{4}}\right) \cos 2 \theta \\
\sigma_{\theta \theta}^{R}= & -p \frac{a^{2}}{r^{2}}+\frac{\sigma_{h 1}+\sigma_{h 2}}{2} \frac{a^{2}}{r^{2}}
\end{aligned}
$$




$$
\begin{gathered}
-\frac{\sigma_{h 1}-\sigma_{h 2}}{2} \frac{3 a^{4}}{r^{4}} \cos 2 \theta \\
\sigma_{r \theta}^{R}=-\frac{\sigma_{h 1}-\sigma_{h 2}}{2}\left(\frac{2 a^{2}}{r^{2}}-\frac{3 a^{4}}{r^{4}}\right) \sin 2 \theta \\
\sigma_{z z}^{R}=-2 v\left(\sigma_{h 1}-\sigma_{h 2}\right) \frac{a^{2}}{r^{2}} \cos 2 \theta
\end{gathered}
$$

et les composantes des contraintes secondaires:

$$
\begin{aligned}
\sigma_{r}^{\mathrm{s}}= & \mathrm{p} \frac{\mathrm{a}^{2}}{\mathrm{r}^{2}}+\frac{\sigma_{h 1}+\sigma_{h 2}}{2}\left(1-\frac{a^{2}}{r^{2}}\right)+\frac{\sigma_{h 1}-\sigma_{h 2}}{2} \\
& \left(1-\frac{4 a^{2}}{r^{2}}+\frac{3 a^{4}}{r^{4}}\right) \cos 2 \theta \\
\sigma_{\theta \theta}^{\mathrm{s}}= & -p \frac{a^{2}}{r^{2}}+\frac{\sigma_{h 1}+\sigma_{h 2}}{2}\left(1+\frac{a^{2}}{r^{2}}\right) \\
& -\frac{\sigma_{h 1}-\sigma_{h 2}}{2}\left(1+\frac{3 a^{4}}{r^{4}}\right) \cos 2 \theta \\
\sigma_{r \theta}^{\mathrm{s}}= & \frac{\sigma_{h 1}-\sigma_{h 2}}{2}\left(-1-\frac{2 a^{2}}{r^{2}}+\frac{3 a^{4}}{r^{4}}\right) \sin 2 \theta \\
\sigma_{z z}^{\mathrm{s}}= & \sigma v-v\left(\sigma_{h 1}-\sigma_{h 2}\right) \frac{2 a^{2}}{r^{2}} \cos 2 \theta
\end{aligned}
$$

De ces dernières formules on obtient les contraintes dans la roche immédiatement après le forage. On rappelle que pour ce cas la contrainte moyenne et la contrainte équivalente sont:

$$
\sigma=\frac{\sigma_{r r}+\sigma_{\theta \theta}+\sigma_{m}}{3}
$$

$\bar{\sigma}^{2}=\sigma_{r \mathrm{r}}^{2}+\sigma_{\theta \theta}^{2}+\sigma_{z z}^{2}-\sigma_{r r} \sigma_{\theta \theta}-\sigma_{\theta \theta} \sigma_{z z}-\sigma_{z z} \sigma_{x}$ $+3 \sigma_{r \theta}^{2}$

et pour les contraintes primaires on obtient:

$$
\begin{gathered}
\sigma^{\mathrm{P}}=\frac{\sigma_{\mathrm{h} 1}+\sigma_{\mathrm{h} 2}+\sigma_{\mathrm{v}}}{3} \\
\left(\tilde{\sigma}^{\mathrm{P}}\right)^{2}=\left(\frac{\sigma_{\mathrm{h} 1}+\sigma_{\mathrm{h} 2}}{2}-\sigma_{\mathrm{v}}\right)^{2}+3\left(\frac{\sigma_{\mathrm{h} 1}-\sigma_{\mathrm{h} 2}}{2}\right)^{2}
\end{gathered}
$$

qui sont indépendantes de l'angle $\theta$.

Pour donner un exemple numérique, considérons de nouveau la loi de comportement pour le granite donnée plus haut. L'état primaire de contrainte comme suggéré par les données de LINDNER et HALPERN (1978) utilisées ici pour le seul but de donner un exemple numérique illustratif, est supposé de la forme suivante:

$$
\sigma_{\mathrm{v}}=0.027 \mathrm{~h}, \sigma_{\mathrm{h} 2}=5.4+0.012 \mathrm{~h}, \sigma_{\mathrm{h} 1}=\mathrm{n \sigma}_{\mathrm{h} 2}
$$

avec $h$ en mètres et les contraintes en $\mathrm{MPa}$. Pour le coefficient $n$ on a utilisé les valeurs suivantes: $n=1$, $n=2, n=4$ et $n=6$. Sur la figure 9 sont montrés

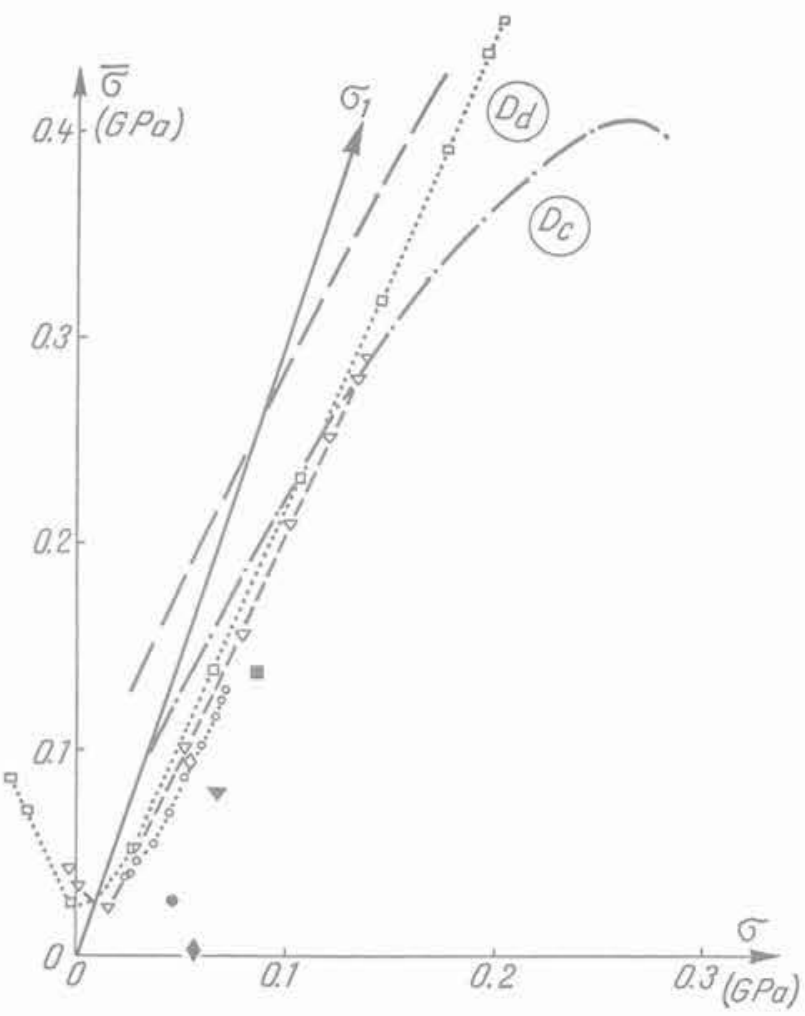

Fig. 9. - Etats des contraintes autour du puits (figures vides) et états primaires (figures pleines).

les résultats pour la profondeur $\mathrm{h}=2 \mathrm{~km}$. Les cercles vides représentent les contraintes pour $\mathrm{r}=\mathrm{a}$ et $\theta=0^{\circ} ; 10^{\circ} ; \ldots ; 90^{\circ}$ obtenues avec $n=2$. L'état primaire de contraintes correspondant a été représenté par un cercle plein. D'une façon similaire les triangles correspondent à $\mathrm{n}=4$ et les carrés à $\mathrm{n}=6$. Sur toutes ces courbes, les points de l'extrême gauche correspondent à $\theta=0^{\circ}$ et ceux de l'extrême droite à $\theta=90^{\circ}$

Considérons maintenant ce que si passe quand $\mathrm{n}$ croît en partant de $n=1$. On observe d'abord que si l'état primaire est hydrostatique $\sigma_{\mathrm{v}}=\sigma_{\mathrm{h} 1}=\sigma_{\mathrm{h} 2}$ cet état de contrainte est représenté par un losange plein sur l'axe Oб. L'état secondaire correspondant est aussi représenté par un losange vide: c'est un point unique indépendant de $\theta$. Ce point a la même contrainte moyenne que celle de l'état primaire correspondant. Dans l'exemple numérique donné ici on a pris $\sigma_{\mathrm{h} 1}=\sigma_{\mathrm{h} 2}=\sigma_{\mathrm{v}}$ et la dernière composante a été obtenue de $(7.7)_{1}$. En conséquence dans ce cas particulier l'état secondaire ne touche pas la frontière compressibilité/dilatance et partant la roche reste dans un état compressible.

Lorsque la valeur de $\mathrm{n}$ croît graduellement, la première fois que la dilatance apparaît le long du contour $r=a$ pour une certaine valeur de $n$, c'est au point $\theta=90^{\circ}$. Si $n$ continue à croître, cette région dilatante s'accroît symétriquement par rapport au point $\theta=90^{\circ}$. Pour des valeurs encore plus grandes de $n$ ou pour des profondeurs plus grandes, l'état secondaire peut même toucher la frontière de rupture (approximativement marquée par une ligne grasse interrompue). Il y a 
deux cas possibles. Après le forage, autour de $r=a$ et pour des valeurs de $\theta$ voisines de $\theta=0^{\circ}$, la roche est dans un état élastique, c'est-à-dire que par forage on produit une décharge. L'état de contrainte secondaire $\sigma^{S_{0}}$ est de traction, c'est-à-dire $\sigma<0$. La composante $\sigma_{\theta \Theta}^{\mathrm{S}}$ peut dépasser en valeur absolue la limite de résistance de la roche en traction pure. Il en résulte que dans une région voisine de $\theta=0^{\circ}$ une rupture est possible en traction. C'est une rupture rapide.

D'autre part dans une région voisine de $\theta=90^{\circ}$ la roche est dilatante et se déforme par fluage ayant tendance à fermer le trou. Pendant ce fluage un dommage de la roche par dilatance se produit lentement en fonction du temps. Une mesure de ce dommage peut être le paramètre $\mathrm{d}$ introduit par (3.10). Quand, pendant le fluage, ce paramètre atteint une valeur critique typique pour la roche considérée, un processus lent de rupture progressive apparaît à $r=a, \theta=90^{\circ}$ et s'élargit en fonction du temps des deux côtés de $\sigma=90^{\circ}$ et pour $r>a$.

Dónc si les deux composantes $\sigma_{\mathrm{h} 1}$ et $\sigma_{\mathrm{h} 2}$ sont très différentes en amplitude, autour du trou de forage on peut avoir une rupture rapide par traction, aussi bien qu'une rupture lente à la suite d'un processus de dilatance par fluage. Le processus décrit plus haut est semblable à une rupture possible par dilatance autour d'un tunnel horizontal décrite par CRISTESCU (1984c, Ch. 12).

\section{CONCLUSIONS}

De tout ce qu'a été dit auparavant on peut arriver à quelques conclusions importantes pour les applications.

Une description mathématique du fluage de la roche autour d'un forage pétrolier ou d'un puits peut être faite si les propriétés de la roche sont étudiées au laboratoire et si une loi de comportement de type élasto/viscoplastique est formulée.

Les domaines autour du puits où la roche devient dilatante ou compressible peuvent être trouvés avec précision si l'état primaire de contrainte est connu. Deux cas sont discutés. Si toutes les contraintes primaires longitudinales sont égales, la dilatance n'apparaît tout autour de la circonférence $r=a$ du puits qu'aux très grandes profondeurs seulement si la roche est dure, et peut apparaître à toutes profondeurs si la roche n'est pas très dure. Dans tous les cas, si la profondeur augmente, le domaine de dilatance autour du puits s'agrandit. Notons aussi que due à la relaxation des contraintes pendant le fluage, la déformation volumique à quelques distances autour de l'orifice est évolutive: au commencement de la déformation par fluage a lieu une dilatance du volume et à la fin de ce processus la roche finit par être compressible,

Mais en général les composantes primaires horizontales ne sont pas égales: il y a une composante horizontale maximale $\sigma_{h 1}$ et une minimale $\sigma_{h 2}$. Dans ce cas la situation est beaucoup plus compliquée. Même aux petites profondeurs il $y$ aura des régions autour du puits où la roche devient dilatante et d'autres où elle est compressible; il est possible aussi d'avoir des régions où la roche est dans un état élastique.

En ce qui concerne la rupture des roches autour des puits, on peut la décrire aussi à l'aide de la loi de comportement si un critère de rupture, ou bien de dommage, est établi. Deux cas sont possibles.

- Si toutes les composantes horizontales des contraintes sont égales, alors une rupture annoncée par une déformation par dilatance très marquée est seulement possible aux grandes profondeurs (grandes valeurs de $\bar{\sigma}$ ).

- Si les composantes primaires horizontales ne sont pas égales, alors la rupture est possible même aux petites profondeurs par deux mécanismes:

- par traction;

- à la suite d'un processus de déformation par fluage produisant la dilatance.

Le premier mécanisme est rapide, lautre est un processus progressif, qui peut arriver après des intervalles de temps assez longs. En tout cas le lieu sur la paroi du puits et le moment où la rupture peut se produire, peuvent être calculés et prédits en utilisant un modèle mathématique.

La grandeur de la différence $\sigma_{h 1}-\sigma_{h 2}$ joue un rôle essentiel en ce qui concerne l'apparition des domaines dilatants et de rupture. En conséquence tout calcul théorique doit être précédé d'une détermination expérimentale de $\sigma_{\mathrm{h} 1}$ et $\sigma_{\mathrm{h} 2}$ dans le lieu particulier en question.

Enfin on ne doit pas oublier que la boue de forage, la température etc. peuvent aussi influencer les phénomènes discutés plus haut.

Remerciements. L'auteur tient à remercier M. P. HA$\mathrm{BIB}$ pour des remarques utiles et Mme C. CRISTESCU pour son aide dans les calculs numériques.

\section{BIBLIOGRAPHIE}

ABOU-SAYED, A.S., BRECHTEL, C.E., CLIFTON, R.J., (1978). - In situ stress determination by hydrofracturing. A fracture mechanics approach. J. Geophys. Res., 83, No B6, pp. 2851-2862.

BRACE, W.F., (1965). - Some new measurements of linear compressibility of rocks, J. Geophys. Res., 70 (2), pp. 391-398.

BRACE, W.F., PAULDING, B.W.Jr., SCHOLZ, C., (1966). - Dilatancy in the fracture of cristalline rocks, J. Geophys. Res., 71, pp. 3939-3953.

BROWN, E.T., HOECK, E., (1978). - Trends in relationships between measured in situ stresses and depth, Int. J. Rock Mech. Min. Sci. and Geomech. Abstr., 15, pp. 211-215.

CRISTESCU, N., (1984a). - Rock plasticity, Sawcuk/ Bianchi, eds., Plasticity Today: Modelling, Methods and Applications, Elsevier Applied Sci. Publ., pp. 643-655. 
CRISTESCU, N., (1984b). - Elastic-viscoplastic constitutive equations for rock-like materials (in press).

CRISTESCU, N., (1984c). - Rock mechanics, University of Bucharest, (in Romanian), 500 p.

CRISTESCU, N., (1985). - Irreversible dilatancy or compressibility of viscoplastic rock-like materials and some applications, Int. J. of Plasticity, 1, 1, (in press).

DREYER, W., (1973). - The science of rock mechanics, part 1, Trans Tech Publ.

HAIMSON, B.C., (1978). - The hydrofracturing stress measuring method and recent field results, Int. J. Rock Mech. Min. Sci, and Geomech. Abstr., 15.

JUMIKIS, A.R., (1979). - Rock mechanics, Trans Tech Publications.

LINDNER, E.N., HALPERN, J.A., (1978). - In situ stress in North America. A Compilation, Int. J. Rock Mech. Min. Sci. and Geomech. Abstr., 15, pp. 183203.
MASSIER, D., CRISTESCU, N., (1981). - In situ creep of rocks, Rev. Roum. Sci. Techn., Ser. Mec. Appl., 26, 5, pp. 687-702.

MASSIER, D., CRISTESCU, N., (1984). - Creep of rocks around horizontal tunnels, (in press).

OBERT, L., DUVALL, W.I., (1967). - Rock mechanics and the design of structures in rock, John Wiley and Sons.

SAXENA, P.C., MOKHASHI, S.L., RAME GOWDA, B.M., (1979). - Rock stress measurements at Nagjhari tunnels, Kalioani hydro-electric project, India, C.R. $4^{e}$ Congrès de la SIMR, Montreux, septembre 1979, vol. 2, pp. 589-594.

VOLAROVITCH, M.P., BAIUK, E.I., LEVYKIN, A.I., TOMASHEVSKAYA, I.S., (1974). - Physicomechanical properties of rocks and minerals at high pressures and temperatures (in Russian), Nauka, Moscow. 